\title{
A new generation of topical chronic wound treatments containing specific MMP inhibitors
}

This article was published in the following Dove Press journal:

Chronic Wound Care Management and Research

4 September 2014

Number of times this article has been viewed

\section{Ravi Shrivastava \\ Nathalie Cucuat \\ Monika Rousse \\ Thomas Weigand \\ Pedro Neto \\ Claire Janicot}

Christiane Shrivastava

VITROBIO Research Institute, Issoire, France

Correspondence: Ravi Shrivastava VITROBIO Research Institute,

ZAC de Lavaur, 63500 Issoire, France

Tel +33473550505

Fax +33473550011

Email vitrobio@orange.fr
Purpose: Incidence of chronic wounds is constantly rising worldwide, but all currently available treatments are intended either to provide symptomatic relief or to assist cicatrization to some extent, but not to directly stimulate cellular growth. Physiologically, chronic wound healing simply requires cell growth to fill the injured cavity. To grow, our cells need to attach onto a cushion, called extracellular matrix (ECM), secreted by the mother cells and composed of multiple proteins. Recent scientific works prove that the concentration of certain matrix metalloproteinases (MMPs) is extremely high in all chronic wounds and, because of their proteolytic nature, some MMPs completely degrade the ECM, hindering cell attachment and cell growth. The aim of this study was to identify, neutralize, and eliminate these MMPs from the wound surface so as to design an effective treatment for chronic wounds.

Methods: Acute and chronic models of human epithelial and fibroblast cells were prepared on a defined ECM cushion in vitro and MMPs were added in the culture medium to identify the MMPs causing ECM disintegration for each cell type. ECM-degrading MMPs were then incubated with selected procyanidin-rich plant extracts (PCDs) and cell growth was reanalyzed.

Results: It was observed that: 1) multiple MMPs are involved in cellular matrix destruction; 2) ECM-destroying MMPs are specific with respect to cell type; and 3) specific PCDs may bind and neutralize selected MMPs.

Conclusion: Topical application of specific plant PCDs to selectively neutralize ECMdestroying MMPs in acute and chronic wounds represents a novel approach for the treatment of superficial and deep skin wounds.

Keywords: extra cellular matrix (ECM), matrix metalloproteinases, procyanidins (PCDs), tannins, ulcers

\section{Introduction}

Global incidence of chronic, nonhealing wounds is on a constant rise. ${ }^{1,2}$ Because of aging populations and progression of vascular diseases and diabetes, in developed countries almost $1 \%-2 \%$ of the population will suffer from chronic wounds in their lifetime, consuming nearly $2 \%-4 \%$ of the total health care budget. ${ }^{3,4}$ Chronic ulcers are also multiplying dramatically in developing countries, notably because of late diagnosis of diabetes. These wounds are extensive, painful, and prone to contamination, and rarely heal. ${ }^{2,5}$

In theory, wound healing should be extremely easy, as it simply requires growth of deeper fibroblast and superficial epithelial cells to fill the wound cavity. In order to grow, those cells must attach onto a cushion serving as framework, called extracellular matrix (ECM), secreted by the specific mother cells and containing multiple proteins 
such as collagen, elastin, fibronectin, integrin, and laminin, and whose composition varies according to cell type. ${ }^{6,7}$ New epithelial and fibroblast daughter cells attach onto their respective ECM to grow, but in absence of ECM, cell attachment is prevented, and as a consequence, cell growth and wound healing are impeded. ${ }^{8,9}$

ECM is specific to each type of cell. Deep wounds affect not only the superficial skin structures but also the underlying muscles, and may reach down to bone tissue. Skin is predominantly composed of epithelial cells with subjacent muscular structures containing fibroblast cells. Deep wound healing therefore requires simultaneous growth of fibroblast and epithelial cells. ${ }^{9,10}$

In spite of the last decades' continuous research, there is currently no truly efficient cell growth-promoting treatment for chronic wounds. ${ }^{11}$ Almost all existing treatments only partially provide the conditions necessary for cell growth. Most are designed to keep the wound surface hydrated, to minimize pain, and reduce the contaminant load, but none is intended to directly stimulate cell growth. ${ }^{12}$ Treatment with ECM components such as hyaluronic acid and collagen, or whole intestinal ECM, has never produced satisfactory results because the composition of naturally secreted ECM is very complex, cell-specific, and practically impossible to reproduce artificially by mixing individual ECM components. ${ }^{8}$

All recent scientific works prove that nonhealing wounds critically lack ECM, a fact directly linked to high concentrations of proteolytic enzymes called matrix metalloproteinases (MMPs). ${ }^{13}$ MMPs comprise a family of over 20 structurally related proteins that are zinc-dependent and calcium-activated endopeptidases, playing a vital role in tissue remodeling, cell migration, cellular regeneration, elimination of excess ECM, and cleavage of unwanted proteins present on the surface of the wound to clean the injured surface and create a favorable ground for healing. ${ }^{14,15}$ Through their enzymatic activity, MMPs may process various types of proteins, including proteases, cytokines, chemokines, growth factors, adhesion molecules, etc, and particularly matrix proteins composing the structure of the ECM, which the present study focuses on. Although the concentration of these MMPs is regulated through tissue inhibitors of metalloproteinases (TIMPs), in chronic wounds the balance between MMPs and TIMPs shifts in favor of MMPs, ${ }^{16,17}$ resulting in excessive degradation of ECM. ${ }^{13,18}$ Those proteolytic enzymes being present in excessively high concentrations in chronic wounds (as high as 65-fold for MMP-1, threefold for MMP-2 proenzyme, sixfold for activated MMP-2, twofold for MMP-8, and 14-fold for MMP-9, compared to average concentrations found in biopsies of traumatic wounds), they end up breaking down not only the unwanted proteins but almost all the ECM constituents as well, which leads to the wound healing process utterly stalling. ${ }^{18-20}$ Therefore, identifying and neutralizing ECM-degrading MMPs is now becoming a major field of scientific research worldwide.

Except for some encouraging in vitro results, ${ }^{21}$ which have led to development of collagen-based wound dressings aiming at broad-spectrum MMP modulation, there is currently no truly promising treatment designed to selectively target and reduce the amount of ECM-damaging MMPs on the wound surface. ${ }^{22}$ This is mostly related to the fact that: 1) the MMPs or the TIMPS participating in ECM destruction are not all identified yet; 2) MMPs are present on the surface of the wound where systemic treatments are not very effective; 3 ) some MMPs are essential to the healing process and should not be inhibited; and 4) multiple MMPs may participate in ECM destruction and it is practically impossible to neutralize the totality of these MMPs with a single specific topical or systemically administered chemical drug.

Therefore, the aim of this study was to first identify all key MMPs involved in the disintegration of epithelial and fibroblast ECMs by employing a new in vitro model of acute and chronic ECM structures of the wound surface. Specific ECM-degrading MMPs were then neutralized by employing plant tannins, inert, nontoxic, and topically nonirritant molecules, that have been found to possess a strong affinity for binding to proteins, so as to design a new topical treatment for chronic wounds.

\section{Materials and methods Preparation of epithelial and fibroblast ECMs}

Live human skin grafts or oral epithelial tissue grafts were obtained from the Department of Vascular Surgery (CHU, Clermont-Ferrand, France), washed with Eagle's Minimum Essential Medium (MEM), cleaned to remove connective tissue and fat, cut into small pieces $\left(1 \mathrm{~cm}^{3}\right)$, and processed further as described by DeQuach et al. ${ }^{23}$ Briefly, tissue was stirred in $1 \%$ (wt/vol) solution of sodium dodecyl sulfate in phosphate-buffered saline (PBS) for 4-5 days, centrifuged, and the pellets were collected and diluted in deionized water (1:10), and finally lyophilized to obtain a fine powder. Before coating the tissue culture plates (24-well, plastic; Corning Incorporated, Corning, NY, USA), $10 \mathrm{mg}$ of matrix was diluted in $1 \mathrm{~mL}$ of pepsin (Sigma-Aldrich Co, St Louis, MO, USA) solution under constant stirring for 48 hours. The matrix was further diluted in $0.1 \mathrm{M}$ acetic acid to 
perfectly mimic skin ECM epithelial or fibroblast tissue matrix. This ECM was used to coat the polystyrene surface for 1 hour at $37^{\circ} \mathrm{C}$, followed by rinsing with PBS.

\section{Human oral primary epithelial cell culture}

Human primary oral epithelial cell cultures (passage 2) were preliminarily purchased from Applied Biological Materials (ABM) Inc. (Richmond, Canada) and stored in liquid nitrogen liquid phase $\left(-196^{\circ} \mathrm{C}\right)$ in a $20 \%$ fetal calf serum (FCS), $10 \%$ dimethyl sulfoxide (DMSO) medium for later use. The cells were initially purchased from ABM Inc, and were then subcultured in our laboratory. To conduct the experiment, cells were thawed $\left(37^{\circ} \mathrm{C}\right.$ for 1 minute), suspended in $5 \mathrm{~mL}$ complete media, centrifuged at 1,500 rpm for 2 minutes to pellet, and the pellet was resuspended in fresh culture medium (TM999) containing 10\% FCS and antibiotics. One milliliter of this cell suspension containing $1 \times 10^{5} \mathrm{cells} / \mathrm{mL}$ was then seeded in each well of the 24-well, epithelial ECM-coated tissue culture plates (Corning Incorporated). The epithelial cells' ECM was particularly rich in collagen with a small proportion of elastin and laminin. Cells were grown in $37^{\circ} \mathrm{C}, 5 \% \mathrm{CO}_{2}$ atmosphere to obtain about $20 \%-30 \%$ cell monolayer within 24 hours, $50 \%-60 \%$ within 72 hours, or $100 \%$ within a week.

\section{Human fibroblast cell cultures}

Normal human skin fibroblast cell line (R2F), closely identical to the granulation tissue cells formed during scar formation, was purchased from CLS Cell Lines Service $\mathrm{GmbH}$ (Eppelheim, Germany) and was initially cultured in $75 \mathrm{~cm}^{2}$ tissue culture flasks (Corning Incorporated) in MEM supplemented with $10 \%$ FCS. Growing cells covered about $20 \%-30 \%$ of the culture surface within 24 hours, between $50 \%$ and $60 \%$ of the surface within 72 hours, and $100 \%$ within 5-6 days of incubation. For experiments, confluent cell monolayers were dispersed with trypsin and a cell suspension containing $1 \times 10^{5}$ cells $/ \mathrm{mL}$ was prepared in an MMP-free MEM containing $10 \%$ FCS and seeded onto the fibroblast cell ECM-coated tissue culture plates.

\section{Acute and chronic cell culture models}

To mimic models of acute and chronic wound environments, 24-hour cell cultures covering approximately $20 \%-30 \%$ of the culture surface with exponentially growing cells were used as acute wound models, while 72-hour cell cultures having $50 \%-60 \%$ cell monolayers were used as chronic wound models. Epithelial cell cultures represented superficial injuries while fibroblast cell cultures were used to mimic chronic wound cells.

\section{Preparation of tannin-rich plant extracts}

Extracts of 145 plants were initially selected to study their effects on the growth of fibroblast and epithelial cells in vitro. Either whole plants or parts of the plant which are traditionally used in phytomedicine were selected for extraction according to the method of Giner-Chavez et al. ${ }^{24}$ In short, initial tannin-rich plant extract was obtained with an aqueous organic solvent containing $70 \%$ acetone and $30 \%$ water. The extracts were then successively passed through Sephadex ${ }^{\circledR}$ LH-20 columns by progressively increasing the volume of methanol $(60 \times 88.5 \mathrm{~cm})$, and the intended fractions were eluted to produce a dry solid. The product was identified by mass spectrometry. The extracts used for experiments contained mainly $60 \%-80 \%$ epicatechin, catechin B1, B2, B3, and C1 fractions. On the basis of initial tannin content $(>20 \%)$, and absence of cytotoxicity or irritation at the maximum concentration that could be used in the final test product, 44 tannin-rich plant extracts (PCDs) were selected after initial screening to be evaluated with regard to their MMP-binding properties. After further testing, only 18 extracts, showing MMP inhibitory activity superior to $10 \%$, were retained. The most effective association of plant PCDs capable of maximally neutralizing selected MMPs was termed "VB-PCDs".

\section{Selection of MMPs involved in ECM destruction}

Based on literature review, all commercially available purified MMPs were tested. Purified recombinant human matrix MMP-1 (intestinal collagenase), MMP-2 (gelatinase), MMP-3 (stromelysin 1, progelatinase), MMP-7 (matrilysin, uterine), MMP-8 (neutrophil collagenase), MMP-9 (gelatinase B), MMP-10 (stromelysin-2), MMP-11 (stromelysin-3), MMP12 (macrophage elastase), MMP-13 (collagenase-3), MMP14 (membrane-inserted/MT1-MMP), MMP-15 (MTMMP2/ SMCP-2), MMP-16 (MMP-X2), MMP-17 (MT4-MMP), MMP-19 (MMP-RASI), MMP-20 (enamelysin), MMP-21, MMP-23 (CA-MMP), MMP-24 (MT5-MMP), MMP-26 (endometase/matrilysin), MMP-27 (MMpeptidase 27), and MMP-28 (epilysin) were purchased from Creative-Biomart (Shirley, NY, USA). All experiments were conducted using a fixed MMP concentration of $10 \mu \mathrm{g} \mathrm{MMP} \mathrm{Eq} / \mathrm{mL}$ (equivalents of solutes per milliliter), which was selected on the basis of the median MMP concentration in chronic wound fluids. ${ }^{25}$ MMPs were then associated with each other to identify the group of epithelial or fibroblast cell matrix-destroying MMPs (VB-MMPs). 


\section{Cell culture treatment procedure}

Plant tannins, MMPs, or tannin-treated MMPs, either alone or in different associations, were preincubated for 1 hour $\left(37^{\circ} \mathrm{C}\right)$ prior to cell culture exposure. One milliliter of test association was introduced in each well of 24-well epithelial or fibroblast cell culture plates corresponding to either acute or chronic models. Cell growth was measured on the seventh day of culture by staining the cells with 3-(4,5-dimethylthiazol-2yl)-2,5-diphenyltetrazolium bromide (MTT) as described by Klebe and Harriss. ${ }^{26}$ The optical density of the MTT solution was determined at $560 \mathrm{~nm}$ using an automatic micro-well reading apparatus (Dynatech MR 400). MMP- and tanninfree cell cultures served as controls. All experiments were conducted in triplicate using six wells per concentration (n $=$ minimum 18 readings per concentration).

\section{Identification of ECM-destroying MMPs}

Initially, MMPs were added individually in the epithelial and fibroblast cell culture mediums and cell growth was compared to the control cultures without any MMP (normal cell growth, with $0 \%$ inhibition). ECM-destroying MMPs, reducing cell growth by more than $10 \%$ compared to the control cultures, were then associated with each other to identify the group of MMPs inducing maximum cell growth inhibition (VB-MMPs).

\section{Evaluation of MMP-neutralizing properties of plant tannins}

To evaluate the capacity of different tannin-rich plant extracts to neutralize MMPs, a fixed concentration of each PCD or VB-PCD ( $5 \mathrm{mg} / \mathrm{mL} / \mathrm{PCD})$, in the form of a PCD powder suspension diluted in MEM, was preincubated for 1 hour with a single MMP or with a defined VB-MMP association (10 $\mu \mathrm{g} / \mathrm{mL} / \mathrm{MMP})$. After 1 hour preincubation, the tanninMMP suspension was applied onto epithelial and fibroblast cell cultures and cell growth was measured. Cell growth was directly proportional to MMP neutralization. Untreated cell cultures served as negative controls while cell cultures treated only with the corresponding MMP served as positive control. All individual plant tannins neutralizing $\mathrm{MMP}(\mathrm{s})$ by at least $25 \%$ were then associated to select the best plant tannin association (VB-PCDs) most capable of counteracting the VB-MMPs inhibitory effect on epithelial or fibroblast cell growth.

\section{Results}

\section{Data analysis}

For in vitro studies, experiments were repeated at least three times, and mean percentage changes in cell number compared to controls are presented as mean \pm standard deviation (SD). The ECM-destroying MMPs (VB-MMPs) specific to epithelial or fibroblast cell types in acute and chronic cell cultures were identified. The tannin-rich plant extracts capable of neutralizing specific groups of VBMMPs in acute models were termed "Proteocyanidins" for epithelial cells and "Mucocyanidins" for fibroblast cells, while VB-PCDs neutralizing chronic model matrixdestroying MMPs were termed "MMP-cyanidins" and "MMP-cynadols", for epithelial and fibroblast cell types, respectively.

\section{Identification of ECM-destroying MMPs}

Among 22 MMPs tested individually, only ten MMPs (MMP-1, -2, -3, -7, -8, -9, -10, -11, -13, and -26) inhibited growth of either epithelial or fibroblast cells in acute or chronic cell cultures by over $25 \%$ compared to the corresponding controls. All other MMPs had much less or no effect on cell growth. Results indicate that MMPs effectively destroy ECM and decrease cell growth and that the activity of MMPs varies according to the cell type and the stage of cell growth. None of the individual MMPs studied in this test inhibited the cell growth totally, indicating that multiple MMPs are involved in ECM destruction. Among the ten ECM-degrading MMPs, MMP-1, -2, -3, -8, -13 and -26 had a stronger effect than the others (Table 1). MMP-2 seems to be particularly involved in the destruction of epithelial cell ECM while MMP-13 degrades mainly the ECM of chronic cell cultures. It is also noted that MMP-8 is particularly active in inhibiting cell growth in its initial phase.

When MMPs were associated with each other, their inhibitory effects on cell growth were neither additive nor synergistic, but maximum cell growth inhibition occurred when more than one MMP was present in the cell culture. Results show that, although those MMPs that destroy epithelial cell ECM also degrade fibroblast cell ECM to some extent, and vice versa, a specific group of MMPs is essential for critical $(>80 \%)$ ECM destruction. During the acute phase of cell growth, MMP-1 and MMP-8 (designated as VB-MMP group 1) destroy mainly epithelial cell ECM and MMPs -1, -3 , and -10 (VB-MMP group 2) destroy mainly fibroblast cell ECM, whereas in chronic cell cultures, MMPs -2, $-3,-9$, and -13 (VB-MMP group 3) affect epithelial cells and MMPs -3, $-9,-13$ and -26 (VB-MMP group 4) fibroblast cells. These groups of MMPs were regarded as the major players in ECM destruction and were used to study the neutralizing effects of specific plant extracts (PCDs). 
Table I Exposure of cell cultures to individual MMPs and MMP associations: inhibitory effect of MMPs on cell growth in vitro

\begin{tabular}{|c|c|c|c|c|}
\hline & \multicolumn{2}{|c|}{$\begin{array}{l}\text { \% reduction in cell growth: acute cell } \\
\text { culture model }\end{array}$} & \multicolumn{2}{|c|}{$\begin{array}{l}\% \text { reduction in cell growth: chronic cell } \\
\text { culture model }\end{array}$} \\
\hline & Epithelial cells & Fibroblast cells & Epithelial cells & Fibroblast cells \\
\hline \multicolumn{5}{|l|}{ MMP } \\
\hline 1 & $53.75( \pm 6.31)$ & $43.56( \pm 6.63)$ & $37.31( \pm 4.52)$ & $34.62( \pm 4.79)$ \\
\hline 2 & $58.69( \pm 5.7 \mathrm{I})$ & $32.54( \pm 5.06)$ & $49.74( \pm 6.4 I)$ & $<25$ \\
\hline 3 & $<25$ & $53.66( \pm 7.59)$ & $48.60( \pm 6.57)$ & $34.85( \pm 5.33)$ \\
\hline 7 & $41.21( \pm 7.15)$ & $<25$ & $34.45( \pm 5.30)$ & $<25$ \\
\hline 8 & $61.48( \pm 8.72)$ & $56.26( \pm 8.83)$ & $38.84( \pm 4.64)$ & $32.65( \pm 4.06)$ \\
\hline 9 & $<25$ & $32.26( \pm 6.39)$ & $36.49( \pm 6.93)$ & $4 I .93( \pm 5.21)$ \\
\hline 10 & $26.82( \pm 5.4 \mathrm{I})$ & $39.47( \pm 4.87)$ & $<25$ & $37.71( \pm 6.88)$ \\
\hline 11 & $36.31( \pm 5.22)$ & $28.34( \pm 4.56)$ & $27.54( \pm 5.21)$ & $34.26( \pm 3.49)$ \\
\hline 13 & $<25$ & $<25$ & $53.22( \pm 6.88)$ & $52.31( \pm 3.58)$ \\
\hline 26 & $31.85( \pm 4.20)$ & $42.55( \pm 8.39)$ & $42.96( \pm 7.44)$ & $55.38( \pm 6.06)$ \\
\hline Others & $<25$ & $<25$ & $<25$ & $<25$ \\
\hline \multicolumn{5}{|l|}{ MMP associations } \\
\hline $1+2$ & $71.53( \pm 9.13)$ & $52.4 I( \pm 5.2 I)$ & $42.36( \pm 4.78)$ & $35.66( \pm 5.69)$ \\
\hline $1+2+3$ & $73.45( \pm 6.32)$ & $61.82( \pm 4.88)$ & $66.45( \pm 5.06)$ & $63.56( \pm 4.20)$ \\
\hline $1+2+8$ & $67.36( \pm 5.56)$ & $54.33( \pm 5.09)$ & $26.74( \pm 5.28)$ & $25.21( \pm 8.12)$ \\
\hline $2+3+9$ & $54.36( \pm 6.03)$ & $42.36( \pm 4.19)$ & $75.86( \pm 6.44)$ & $39.45( \pm 6.25)$ \\
\hline $2+3+12$ & $49.56( \pm 4.57)$ & $47.53( \pm 4.86)$ & $64.30( \pm 6.31)$ & $46.28( \pm 5.82)$ \\
\hline $2+3+13$ & $56.77( \pm 4.91)$ & $51.36( \pm 6.74)$ & $68.08( \pm 5.46)$ & $51.14( \pm 4.64)$ \\
\hline $3+9+13$ & $61.65( \pm 5.18)$ & $45.66( \pm 3.56)$ & $71.34( \pm 6.07)$ & $41.59( \pm 5.47)$ \\
\hline $1+7+12$ & $55.62( \pm 5.65)$ & $51.04( \pm 7.25)$ & $34.56( \pm 3.47)$ & $48.87( \pm 4.59)$ \\
\hline $1+3$ & $67.45( \pm 4.28)$ & $47.34( \pm 6.01)$ & $29.54( \pm 3.98)$ & $31.25( \pm 4.33)$ \\
\hline $1+3+10$ & $68.36( \pm 9.61)$ & $79.89( \pm 4.28)$ & $60.05( \pm 5.56)$ & $72.30( \pm 9.15)$ \\
\hline $1+8$ & $82.31( \pm 6.64)$ & $67.11( \pm 4.55)$ & $71.33( \pm 7.33)$ & $63.33( \pm 6.16)$ \\
\hline $1+8+10$ & $74.12( \pm 5.08)$ & $61.35( \pm 6.40)$ & $63.25( \pm 5.74)$ & $59.89( \pm 4.57)$ \\
\hline $1+10$ & $57.63( \pm 5.37)$ & $71.25( \pm 8.11)$ & $45.08( \pm 5.05)$ & $49.50( \pm 3.11)$ \\
\hline $1+3+9$ & $62.87( \pm 6.40)$ & $56.91( \pm 5.66)$ & $58.11( \pm 5.3)$ & $55.74( \pm 4.09)$ \\
\hline $3+9+10$ & $45.51( \pm 6.03)$ & $42.11( \pm 3.84)$ & $61.04( \pm 5.3)$ & $38.41( \pm 5.74)$ \\
\hline $2+9+10$ & $45.31( \pm 4.44)$ & $36.52( \pm 3.4 I)$ & $59.42( \pm 5.3)$ & $27.54( \pm 2.32)$ \\
\hline $2+9+13$ & $67.91( \pm 4.06)$ & $48.60( \pm 3.26)$ & $72.30( \pm 5.3)$ & $28.09( \pm 3.10)$ \\
\hline $1+9+13$ & $60.44( \pm 6.44)$ & $47.01( \pm 5.28)$ & $68.05( \pm 5.3)$ & $35.51( \pm 3.54)$ \\
\hline $1+10+13$ & $59.45( \pm 5.36)$ & $72.80( \pm 6.09)$ & $33.65( \pm 5.3)$ & $38.07( \pm 3.75)$ \\
\hline $3+10+13$ & $46.91( \pm 5.47)$ & $31.36( \pm 4.33)$ & $40.09( \pm 5.3)$ & $46.25( \pm 6.70)$ \\
\hline $2+3+9$ & $70.11( \pm 6.02)$ & $51.06( \pm 4.27)$ & $72.12( \pm 5.3)$ & $61.39( \pm 8.45)$ \\
\hline $2+3+13$ & $67.64( \pm 7.85)$ & $56.10( \pm 5.30)$ & $71.44( \pm 5.3)$ & $57.78( \pm 7.22)$ \\
\hline $2+3+9+13$ & $72.36( \pm 5.50)$ & $63.72( \pm 5.87)$ & $91.6( \pm 5.3)$ & $67.34( \pm 5.47)$ \\
\hline $3+9+13$ & $67.30( \pm 5.45)$ & $64.35( \pm 6.07)$ & $68.63( \pm 5.3)$ & $65.55( \pm 5.38)$ \\
\hline $3+13+26$ & $62.74( \pm 7.14)$ & $53.21( \pm 5.36)$ & $57.24( \pm 5.3)$ & $53.54( \pm 4.95)$ \\
\hline $3+9+13+26$ & $66.4 I( \pm 4.31)$ & $68.03( \pm 7.13)$ & $71.54( \pm 5.3)$ & $89.21( \pm 7.43)$ \\
\hline $2+3+9+13+26$ & $68.32( \pm 4.73)$ & $70.23( \pm 7.09)$ & $76.35( \pm 5.3)$ & $65.26( \pm 6.85)$ \\
\hline $1+3+9+13+26$ & $61.36( \pm 5.12)$ & $56.47( \pm 4.83)$ & $54.32( \pm 5.3)$ & $59.39( \pm 4.12)$ \\
\hline $2+3+8+13+26$ & $73.35( \pm 6.96)$ & $63.25( \pm 5.05)$ & $71.22( \pm 5.3)$ & $58.78( \pm 4.85)$ \\
\hline Others & $<25$ & $<25$ & $<25$ & $<25$ \\
\hline
\end{tabular}

Notes: Results are expressed as mean inhibition \% ( \pm standard deviation) compared to MMP-free control cultures. Results are shown for only those individual MMPs or MMP associations capable of inhibiting epithelial or fibroblast cell growth by over $25 \%$ as compared to corresponding MMP-free controls. All values are statistically significant ( $P<0.05)$. Abbreviation: MMP, matrix metalloproteinase.

\section{Efficacy of MMP-inhibiting PCDs}

The results of PCD-MMP binding shown in Table 2 indicate that only 18 out of 44 individual PCDs tested had slight-tomoderate MMP neutralization capacity (active PCDs) but all other PCDs were considered inactive as cell growth was not improved by more than $10 \%$. The specific PCD-rich extracts of Camellia sinensis leaves (Cc), Vaccinium macrocarpon fruits (Vma), Vaccinium myrtillus fruit (Vmy), and Vitis vinifera seeds $(\mathrm{Vv})$ exerted stronger MMP inhibition compared to other extracts but none of the individual extracts 
Table 2 Neutralizing effect of tannin-rich plant extracts on MMPs' inhibition of cell growth

\begin{tabular}{|c|c|c|c|c|}
\hline \multirow{2}{*}{$\begin{array}{l}\text { Plant extract or } \\
\text { association of plant } \\
\text { extracts }\end{array}$} & \multicolumn{2}{|c|}{$\%$ cell growth: acute cell culture model } & \multicolumn{2}{|c|}{ \% cell growth: chronic cell culture model } \\
\hline & $\begin{array}{l}\text { VB-MMP group I- } \\
\text { exposed epithelial cells }\end{array}$ & $\begin{array}{l}\text { VB-MMP group 2- } \\
\text { exposed fibroblast cells }\end{array}$ & $\begin{array}{l}\text { VB-MMP group 3- } \\
\text { exposed epithelial cells }\end{array}$ & $\begin{array}{l}\text { VB-MMP group 4- } \\
\text { exposed fibroblast cells }\end{array}$ \\
\hline Negative control (PCDs only) & $96.45( \pm 4.32)$ & $89.52( \pm 8.56)$ & $97.86( \pm 3.02)$ & $87.88( \pm 4.22)$ \\
\hline Positive control (MMPs only) & $6.38( \pm 4.56)$ & $8.45( \pm 3.5 \mathrm{I})$ & $6.68( \pm 2.74)$ & $9.39( \pm 3.86)$ \\
\hline \multicolumn{5}{|c|}{ Preincubation with an individual plant extract (MMPs + I PCD) } \\
\hline Ep & $16.31( \pm 5.40)$ & $9.32( \pm 5.69)$ & $14.79( \pm 4.65)$ & II.78 ( $\pm 4.7 \mathrm{I})$ \\
\hline Mt & $7.06( \pm 3.5 \mathrm{I})$ & $8.11( \pm 2.15)$ & $15.24( \pm 2.64)$ & $12.58( \pm 4.11)$ \\
\hline $\mathrm{Cc}$ & $45.34( \pm 7.90)$ & $24.12( \pm 4.18)$ & $42.36( \pm 6.04)$ & $33.68( \pm 5.79)$ \\
\hline Ac & $21.46( \pm 5.54)$ & $|9.3|( \pm 3.84)$ & I $3.74( \pm 4.88)$ & $21.69( \pm 2.22)$ \\
\hline Vma & $31.25( \pm 4.77)$ & $29.47( \pm 3.79)$ & $19.87( \pm 3.22)$ & $27.39( \pm 6.58)$ \\
\hline $\mathrm{Gb}$ & II.36 ( \pm 3.74$)$ & I $7.55( \pm \mathrm{I} .56)$ & $12.89( \pm 2.12)$ & $18.77( \pm 3.53)$ \\
\hline Vmy & $40.77( \pm 5.42)$ & $43.87( \pm 6.49)$ & $39.62( \pm 8.08)$ & $29.39( \pm 5.05)$ \\
\hline $\mathrm{Pg}$ & $15.87( \pm 3.45)$ & $23.59( \pm 1.87)$ & $28.4 I( \pm 4.03)$ & $24.78( \pm 3.34)$ \\
\hline$V_{v}$ & $22.43( \pm 3.57)$ & $19.50( \pm 2.31)$ & $45.32( \pm 7.12)$ & $41.10( \pm 5.45)$ \\
\hline Asc & $8.69( \pm 3.85)$ & $19.34( \pm 4.15)$ & $13.74( \pm 2.58)$ & $27.56( \pm 4.85)$ \\
\hline $\mathrm{Sa}$ & $12.74( \pm 3.16)$ & 16.49 & $8.4 \mathrm{I}( \pm 2.47)$ & $17.58( \pm 3.56)$ \\
\hline Sn & $16.37( \pm 5.12)$ & $19.87( \pm 3.70)$ & $22.19( \pm 3.56)$ & $20.11( \pm 4.27)$ \\
\hline $\mathrm{Rn}$ & $26.31( \pm 9.21)$ & $18.34( \pm 2.89)$ & $24.05( \pm 5.36)$ & $17.14( \pm 4.54)$ \\
\hline Cs & $11.39( \pm 3.78)$ & $26.70( \pm 5.77)$ & $15.09( \pm 2.57)$ & $31.06( \pm 3.65)$ \\
\hline Qac & $21.87( \pm 3.55)$ & $24.14( \pm 5.69)$ & $12.82( \pm 3.54)$ & $16.5 \mid( \pm 4.08)$ \\
\hline Qin & $15.54( \pm 5.42)$ & $24.25( \pm 4.26)$ & $19.87( \pm 3.68)$ & $22.49( \pm 3.08)$ \\
\hline Tch & $27.36( \pm 4.07)$ & $25.45( \pm 3.35)$ & $19.08( \pm 3.11)$ & $17.70( \pm 4.10)$ \\
\hline Gmx & || $.5 \mid( \pm 3.3 \mid)$ & $8.27( \pm 2.11)$ & $21.36( \pm 6.02)$ & $26.31( \pm 5.07)$ \\
\hline Others & $<10$ & $<10$ & $<10$ & $<10$ \\
\hline \multicolumn{5}{|c|}{ Preincubation with associations of plant extracts (MMPs + PCDs) } \\
\hline $\mathrm{Cc}+\mathrm{Vmy}$ & $71.78( \pm 5.45)$ & $91.56( \pm 7.58)$ & $68.90( \pm 8.05)$ & $94.56( \pm 7.58)$ \\
\hline $\mathrm{Cc}+\mathrm{Vv}_{\mathrm{v}}$ & $69.26( \pm 5.60)$ & $74.12( \pm 5.20)$ & $86.95( \pm 6.31)$ & $76.39( \pm 6.89)$ \\
\hline$V m a+V m y$ & $93.63( \pm 8.24)$ & $72.44( \pm 6.04)$ & $89.96( \pm 7.80)$ & $74.91( \pm 6.66)$ \\
\hline $\mathrm{Vma}+\mathrm{Sn}$ & $82.01( \pm 8.10)$ & $67.00( \pm 4.11)$ & $72.10( \pm 7.48)$ & $64.20( \pm 6.52)$ \\
\hline$V_{v}+R n$ & $58.23( \pm 6.4 I)$ & $87.64( \pm 7.26)$ & $69.45( \pm 7.36)$ & $87.09( \pm 8.11)$ \\
\hline $\mathrm{Ac}+\mathrm{Cc}$ & $68.98( \pm 5.48)$ & $45.78( \pm 7.16)$ & $71.55( \pm 5.10)$ & $51.56( \pm 5.35)$ \\
\hline$A c+C s$ & $48.66( \pm 4.78)$ & $73.45( \pm 7.10)$ & $56.70( \pm 6.5 \mathrm{I})$ & $76.64( \pm 5.90)$ \\
\hline$V m y+G b$ & $73.11( \pm 7.68)$ & $46.38( \pm 5.09)$ & $70.15( \pm 6.80)$ & $56.45( \pm 3.85)$ \\
\hline$V m a+G b$ & $66.40( \pm 4.68)$ & $69.40( \pm 6.54)$ & $54.79( \pm 7.89)$ & $61.60( \pm 8.48)$ \\
\hline$A c+Q a c$ & $57.88( \pm 4.70)$ & $41.70( \pm 3.33)$ & $54.28( \pm 5.36)$ & $47.56( \pm 5.47)$ \\
\hline$A c+$ Qin & $66.41( \pm 4.78)$ & $75.01( \pm 3.17)$ & $41.09( \pm 4.55)$ & $36.57( \pm 5.22)$ \\
\hline$A c+S n$ & $46.78( \pm 6.50)$ & $71.10( \pm 6.01)$ & $54.09( \pm 6.30)$ & $73.80( \pm 5.29)$ \\
\hline $\mathrm{Sn}+\mathrm{Qac}$ & $62.61( \pm 6.32)$ & $46.87( \pm 3.96)$ & $63.12( \pm 7.25)$ & $49.55( \pm 4.4 I)$ \\
\hline Sn + Qin & $72.33( \pm 4.38)$ & $65.91( \pm 6.63)$ & $70.02( \pm 7.00)$ & $66.33( \pm 4.33)$ \\
\hline$A c+G b+V m a$ & $36.35( \pm 5.55)$ & $40.19( \pm 6.70)$ & $72.45( \pm 8.13)$ & $49.60( \pm 4.99)$ \\
\hline$A c+G b+V m y$ & $81.20( \pm 7.77)$ & $56.40( \pm 5.07)$ & $69.22( \pm 7.17)$ & $65.28( \pm 7.12)$ \\
\hline$A c+Q a c+Q i n$ & $55.65( \pm 7.16)$ & $69.60( \pm 4.15)$ & $66.29( \pm 8.44)$ & $59.87( \pm 4.94)$ \\
\hline $\mathrm{Cc}+\mathrm{Vma}_{\mathrm{a}}+\mathrm{Vv}_{\mathrm{v}}$ & $83.54( \pm 8.46)$ & $73.49( \pm 5.25)$ & $84.29( \pm 7.30)$ & $81.46( \pm 6.56)$ \\
\hline$C c+V_{v}+S n$ & $88.18( \pm 8.06)$ & $72.45( \pm 6.68)$ & $86.06( \pm 7.32)$ & $72.10( \pm 7.58)$ \\
\hline$S n+V v+G m x$ & $76.01( \pm 6.60)$ & $64.49( \pm 7.37)$ & $67.90( \pm 5.65)$ & $64.11( \pm 7.03)$ \\
\hline$C c+V v+S n+V m a$ & $72.46( \pm 8.00)$ & $82.64( \pm 4.97)$ & $73.39( \pm 7.10)$ & $68.90( \pm 4.88)$ \\
\hline$C c+V v+S n+V m y$ & $75.31( \pm 7.77)$ & $61.73( \pm 4.90)$ & $76.44( \pm 8.02)$ & $71.06( \pm 5.7 I)$ \\
\hline Others & $<40$ & $<40$ & $<40$ & $<40$ \\
\hline
\end{tabular}

Notes: The selected groups of MMPs (VB-MMP group I, VB-MMP group 2, VB-MMP group 3, VB-MMP group 4) were preincubated with individual PCDs to select MMPneutralizing PCDs. Active PCDs were then associated with each other to find the PCD associations capable of inducing maximum MMP neutralization in the acute and chronic cultures of epithelial and fibroblast cells. Cell growth was considered proportional to MMP neutralization. Cell cultures treated only with PCDs were considered as negative cell cultures and those treated only with MMPs as positive cell cultures. Results are presented as mean \% cell growth ( \pm standard deviation) of three individual experiments ( $n=6$ wells for each experiment). All values are statistically significant $(P<0.05)$ compared to the corresponding control values.

Abbreviations: MMP, matrix metalloproteinase; VB-MMP groups, defined MMP associations identified by Vitrobio for their specific involvement in ECM degradation; ECM, extracellular matrix; PCD, procyanidin-rich plant extracts; Ep, Echinacea purpurea plant; Mt, Mimosa tenuiflora bark; Cc, Camellia sinensis leaves; Ac, Acacia catechu gall; Vma, Vaccinium macrocarpon fruits; Gb, Gingko biloba leaves; Vmy, Vaccinium myrtillus fruit; Pg, Punica granatum rind; Vv, Vitis vinifera seeds; Asc, Aesculus hippocastanum seeds; Sa, Salix alba plant; Sn, Sambucus nigra fruits; Rn, Ribes nigrum fruit; Cs, Cedrela sinensis root; Qac, Quercus acutissima fruits; Qin, Quercus infectoria gall; Tch, Terminalia chebula fruits; Gmx, Glycine max seed. 
was capable of neutralizing all the MMPs, as cell growth was never higher than $45 \%$ in any of the cultures. The Cc extract was particularly active on the epithelial cells while Vv extract exhibited higher MMP inhibition in chronic epithelial and fibroblast cell culture models.

When active PCDs were associated with each other, MMP inhibition was markedly increased, proving that a few tannin-rich plant extracts effectively bind and thus neutralize selective MMPs and that more than one PCD is required to neutralize the entire group of epithelial or fibroblast, acute or chronic, ECM-degrading MMPs. The association does not produce an additive neutralizing effect, but nearly all the PCDs retain their individual MMP-binding capacity. The associations of $\mathrm{Cc}+\mathrm{Vmy}, \mathrm{Cc}+\mathrm{Vv}, \mathrm{Vma}+\mathrm{Vmy}, \mathrm{Cc}+$ $\mathrm{Vma}+\mathrm{Vv}$, and $\mathrm{Cc}+\mathrm{Vv}+$ Sambucus nigra fruits (Sn) were highly active as preferred MMP inhibitors. Vma $+\mathrm{Sn}$ and $\mathrm{Vmy}+$ Gingko biloba leaves showed higher binding affinity for the MMP group responsible for epithelial cell ECM degradation, whereas Acacia catech $u$ gall + Cedrela sinensis root or Acacia catechu gall + Sn had more affinity for those affecting fibroblast ECM. Interestingly, associating more than three active PCDs such as $\mathrm{Cc}+\mathrm{Vv}+\mathrm{Sn}+\mathrm{Vma}$ or $\mathrm{Cc}+$ $\mathrm{Vv}+\mathrm{Sn}+\mathrm{Vmy}$ does not seem to further enhance MMP neutralization: on the contrary, cell growth was slightly less when using these combinations of PCDs, indicating that PCDs are selective with respect to MMP binding and must be meticulously chosen based on the precise group of MMPs to be neutralized.

\section{Discussion}

The prevalence of chronic wounds is increasing around the world, owing principally to aging, often bedridden, populations in developed countries and to the rapid progression of obesity and diabetes predisposing to diabetic and venous leg ulcers in developing countries. ${ }^{27}$

Because those types of wounds and ulcers usually afflict already debilitated patients, and since the majority of the currently available treatments require follow-up by medical experts, very often those patients must remain in the hospital to be monitored, with their hospital stay actually costing much more than the treatment itself.

In theory, any injury to the body's surface should heal rapidly as lesion repair simply necessitates filling the injured surface with identical cellular structures: epithelial cells, or fibroblast cells in case of deeper injuries for which it is more difficult to restore the normal physiological tissue structure in the injured cavity. ${ }^{10}$ In chronic wounds, however, severe degradation of the ECM scaffolding essential to cell growth and tissue repair is the fundamental cause of the halted healing. The normal healing process begins immediately after the injury occurs, with the formation of a blood clot, followed by local inflammation, liberation of growth factors (particularly PDGF [platelet-derived growth factor], EGF [epithelial growth factor], and FGF [fibroblast growth factor]), activation of the body's physiological wound surface cleaning mechanism through the liberation of multiple MMPs to break down waste protein molecules, cell migration and proliferation in a clean environment, and finally tissue remodeling with normal cellular structure or with scar tissue. ${ }^{28,29}$ Depending on the extent and depth of the injury, this process may take from a few days up to a few weeks to achieve complete healing. ${ }^{30}$ Once the injured surface is clean and free of toxins, chemicals, and contaminants, the growth of epithelial cells, on the surface, and fibroblast cells, in underlying tissue layers, may resume and progressively reduce the circumference and depth of the wound simultaneously.

New tissue structures must affix onto a surface, and newly generated cells need to attach onto a kind of cushion to reach maturation, divide, and, by perpetuating this cycle, carry the healing process through. This cushion is provided by the ECM, which is specific to each type of cell, the mother cells each secreting their own ECM components which then assemble on the recovering tissue surface so as to supply the new and growing cells with a fresh ECM bed for attachment. This is why in vitro cell cultures require specific ECM-treated tissue culture plates to grow each type of cell; even so, it may still be difficult to grow certain cells (such as liver epithelial cells) in vitro as their ECM cannot be synthesized artificially. ${ }^{31}$

The MMPs secreted into the wound cavity by each type of cell also vary according to the location, as well as severity and chronicity, of the lesion. It has now been clearly established that the concentration of those MMPs in chronic wounds is way too high in chronic sores and ulcers, ${ }^{32}$ leading to destruction of the $\mathrm{ECM}^{33,34}$ and delayed healing. ${ }^{35}$ Although MMPs have no effect on cellular maturation and division, they utterly block the healing process by disintegrating the proteins composing the ECM, thereby preventing the attachment of the new daughter cells. As a consequence, the wound cavity remains unfilled and open for months or even years, continuously exposed to external contaminants, a situation that keeps the body pouring ever more MMPs into the gaping lesion to clean it of loose proteinous debris, foreign material, and contaminants. The major findings of this study are that not all but only certain MMPs are involved in the destruction of ECM, and that the absence of cellular growth in vitro and 
a halted healing process in vivo may be related to a lack of ECM. Because the concentration of MMPs is extremely high in chronic, nonhealing wounds, and because cellular growth is fundamental for wound healing, our findings also prove that the absence of ECM may be a primary cause of wounds having become chronic.

In the absence of precise knowledge regarding the role of each MMP in ECM degradation or in wound repair, none of the currently available treatments is designed to directly counteract this basic phenomenon responsible for hindering or completely blocking the healing process. ${ }^{34,36}$ Finding new MMP-inhibiting treatments is a tricky challenge for the pharmaceutical industries as many MMPs are involved and they may not all be blocked by using a single chemical or biological molecule, ${ }^{37}$ although some antibiotics such as doxycycline and minocycline have been shown to improve wound healing through immunomodulatory and anti-inflammatory activity, but they are not directed to antagonize all the ECM-degrading MMPs. ${ }^{38,39}$ Furthermore, some MMPs are essential for cellular migration and tissue modeling and therefore cannot be blocked without adversely affecting the repair process. ${ }^{40}$ Lastly, because MMPs are present on the surface of the injury, only topical MMP inhibitors can adequately accomplish this delicate function, with the added advantage of lack of systemic toxicity. These limitations were so important that, in spite of recognizing the dire need to find MMP inhibitors to treat wounds, ${ }^{25,36}$ scientific research groups worldwide shied away from launching a program to precisely identify, and then neutralize, the ECM-degrading MMPs in order to conceive an effective treatment. Ongoing research consists mostly of developing fancy bandages to protect, hydrate, or absorb MMPs directly from the wound surface; ${ }^{37}$ discovering new antiseptics or applying vacuum pressure to suck MMPs from the wound; ${ }^{40}$ using artificial or natural tissue homogenate ECM or ECM components; ${ }^{41}$ growth factors for topical application; genetically engineered skin grafts, etc, but, to date, none of these treatments have shown any truly encouraging results, although attempts at broad-spectrum MMP modulation have been shown to assist wound healing to some extent. . $^{21,42}$

The results of this study clearly prove that MMPs degrade the ECM, that multiple MMPs are involved in this process, and that there are specific MMPs corresponding to different types of lesions. ${ }^{31}$ Although some MMPs can be common to several wound types, different MMP associations are involved in ECM destruction according to the composition of the ECM, which depends on the types of cells and the stage of cellular growth.
MMPs are proteins with variable structures, and tannins are known to bind and precipitate specific proteins. The protein-binding properties of specific tannins have already been demonstrated by Shrivastava, ${ }^{43,44}$ and specific tannins have been employed to neutralize enzymes, ${ }^{45}$ virus glycoproteins,${ }^{46,47}$ or bacterial biofilm proteins. ${ }^{48}$ To avoid possible toxicity and overcome the specificity of synthetic tannins, we used tannin-rich plant extracts that mainly contained the procyanidin fraction of plant polyphenols, and sought the PCD association exhibiting the highest capability to bind with the selected group of ECM-degrading MMPs. Furthermore, tannins present the advantage of being big molecules, which cannot penetrate the cells, have no interaction with cellular structures, and are known to be nonirritant and nontoxic to cells. ${ }^{49}$ Preincubation of ECM-destroying MMPs with those PCDs led to an enhanced cellular growth, reflecting the fact that tannins neutralize MMPs and consequently prevent ECM destruction. Inhibiting not all but only certain MMPs permitted restoring normal cellular growth, demonstrating that a specific group of MMPs was responsible for the destruction of ECM.

The fact that barely $10 \%$ of tannins possess sufficient capabilities to bind with MMPs, the necessity of associating different tannins to block a group of MMPs, and the observation that a determined group of PCDs was necessary to inhibit completely those MMPs so as to restore normal cell growth, together prove that tannin-MMP binding is specific. Tannins or PCDs, known for their affinity for proteins, have a complex chemical structure and therefore can probably establish linkage to several MMPs at the same time. But the necessity to introduce three or four PCDs to obtain complete MMP neutralization indicates that even though one PCD is capable of multiple binding, it cannot bind to all MMPs implicated and consequently different PCDs are necessary to ensure all targeted MMPs are neutralized.

Furthermore, it is noteworthy that the results of MMP neutralization by PCDs were neither additive nor synergistic, but even slightly antagonistic in some particular associations; this indicates that probably two different PCDs are competing with each other for MMP binding, or that they may interact with each other, leading, in either eventuality, to a lesser activity. The tannins must therefore be selected and precisely associated according to the desired resulting activity and also to the type, chronicity, and location of the wound to be treated. PCDs can be used in the form of a fine powder or applied as a cream or liquid on the wound surface. Proteocyanidins can be used to treat acute epithelial injuries such as oral ulcers; Mucocyanidins can be applied 
on acute deep wounds such as oral mucositis ulcers; MMPcyanidins are especially suited to neutralizing superficial chronic wounds such as skin fissures; and MMP-cynadols to treating deep chronic injuries involving destruction of both epithelial and fibroblast cells as in pressure ulcers, diabetic wounds, and venous leg ulcers. Further studies are required, however, to determine whether different types of MMPs are present in other types of wounds, and whether different PCD compositions or concentrations are necessary to treat different chronic wounds such as diabetic ulcers, pressure ulcers, and venous ulcers.

\section{Conclusion}

The results of this study confirm that MMP-induced disintegration of ECM is the primary factor hindering the wound healing process, where not all but only a few specific MMPs destroy the ECM. As ECM is essential for cell growth and wound healing, and as ECM cannot be synthesized due to its complex composition, ${ }^{50}$ one of the best approaches to treating chronic wounds and injuries is to stop MMP-induced ECM degradation. Ongoing experiments undertaken in our laboratory show that the type and concentration of MMPs vary between acute and chronic oral ulcers, but the MMPs in pressure ulcers, diabetic ulcers, and venous leg ulcers are more or less identical. The ultimate objective of this study was the novel identification of specific tannins or tannin fractions to antagonize the MMPs which have been determined to be corrosive to the ECM scaffold, and thus are implicated in delayed or halted wound repair process, so as to design a new generation of treatment with which to heal chronic wounds. Initial clinical results incorporating selective MMP-cynadols in an osmotically active filmogen solution clearly showed extraordinary rapid healing potential of the product on severe nonhealing chronic wounds. ${ }^{10}$

Treating wounds by topical application of specific MMP antagonists in order to preserve the ECM opens up completely new terrain for conceiving an effective treatment promoting the healing of chronic wounds.

\section{Acknowledgment}

This study was sponsored by the VITROBIO Research Institute.

\section{Disclosure}

This study was sponsored by the VITROBIO Research Institute. The authors report no conflicts of interest in this work. The authors' unique purpose was to make their findings public, so as to disseminate scientific knowledge of important significance to wound care and management. This product and the corresponding patents are the properties of the VITROBIO Research Institute.

\section{References}

1. Spear M. Acute or chronic? What's the difference? Plast Surg Nurs. 2013;32(2):98-100.

2. Sen CK, Gordillo GM, Roy S, et al. Human skin wounds: a major and snowballing threat to public health and the economy. Wound Repair Regen. 2009;17(6):763-771.

3. Jones KR, Fennie K. Factors influencing pressure ulcer healing in adults over 50: an exploratory study. J Am Med Dir Assoc. 2007;8(6): 378-387.

4. Baumgarten M, Margolis DJ, Localio AR, et al. Pressure ulcers among elderly patients early in the hospital stay. J Gerontol A Biol Sci Med Sci. 2006;61(7):749-754.

5. de Meneses LC, Blanes L, Francescato Veiga D, Carvalho Gomes H, Masako Ferreira L. Health-related quality of life and self-esteem in patients with diabetic foot ulcers: results of a cross-sectional comparative study. Ostomy Wound Manage. 2011;57(3):36-43.

6. Droz D, Patey N, Paraf F, Chrétien Y, Gogusev J. Composition of extracellular matrix and distribution of cell adhesion molecules in renal cell tumors. Lab Invest. 1994;71(5):710-718.

7. Pilcher BK, Gaither-Ganim J, Parks WC, Welgus HG. Cell type-specific inhibition of keratinocyte collagenase-1 expression by basic fibroblast growth factor and keratinocyte growth factor. A common receptor pathway. J Biol Chem. 1997;272(29):18147-18154.

8. Chen AK, Delrio FW, Peterson AW, Chung KH, Bhadiraju K, Plant AL. Cell spreading and proliferation in response to the composition and mechanics of engineered fibrillar extracellular matrices. Biotechnol Bioeng. 2013;110(10):2731-2741

9. Stekelenburg A, Gawlitta D, Bader DL, Oomens CW. Deep tissue injury: how deep is our understanding? Arch Phys Med Rehabil. 2008;89(7): $1410-1413$.

10. Shrivastava R. Clinical evidence to demonstrate that simultaneous growth of epithelial and fibroblast cells is essential for deep wound healing. Diabetes Res Clin Pract. 2011;1(92):92-99.

11. Bjarnsholt T, Kirketerp-Møller K, Jensen PØ, et al. Why chronic wounds will not heal: a novel hypothesis. Wound Repair Regen. 2008;16(1): $2-10$.

12. Chin KY, Anandan SM, Koshal K, Gujadhur P. Current and future developments in the treatment of chronic wounds. Open Access Surgery. 2013;6:43-53.

13. Martins VL, Caley M, O'Toole EA. Matrix metalloproteinases and epidermal wound repair. Cell Tissue Res. 2013;351(2):255-268.

14. Newby AC. Matrix metalloproteinases regulate migration, proliferation, and death of vascular smooth muscle cells by degrading matrix and non-matrix substrates. Cardiovasc Res. 2006;69(3):614-624.

15. Mott JD, Werb Z. Regulation of matrix biology by matrix metalloproteinases. Curr Opin Cell Biol. 2004;16(5):558-564.

16. Muller M, Trocme C, Lardy B, Morel F, Halimi F, Benhamou PY. Matrix metalloproteinases and diabetic foot ulcers: the ratio of MMP-1 to TIMP-1 is a predictor of wound healing. Diabet Med. 2008;25(4): 419-426.

17. Moali C, Hulmes DJ. Extracellular and cell surface proteases in wound healing: new players are still emerging. Eur J Dermatol. 2009;19(6): $552-564$.

18. Lobmann R, Ambrosch A, Schultz G, Waldmann K, Schiweck S, Lehnert H. Expression of matrix-metalloproteinases and their inhibitors in the wounds of diabetic and non-diabetic patients. Diabetologia. 2002;45(7):1011-1016.

19. McCarty SM, Cochrane CA, Clegg PD, Percival SL. The role of endogenous and exogenous enzymes in chronic wounds: a focus on the implications of aberrant levels of both host and bacterial proteases in wound healing. Wound Repair Regen. 2012;20(2):125-136. 
20. Pirilä E, Korpi JT, Korkiamäki T, et al. Collagenase-2 (MMP-8) and matrilysin-2 (MMP-26) expression in human wounds of different etiologies. Wound Repair Regen. 2007;15(1):47-57.

21. Cullen B, Watt PW, Lundqvist C, et al. The role of oxidized regenerated cellulose/collagen in chronic wound repair and its potential mechanism of action. Int J Biochem Cell Biol. 2002;34(12):1544-1556.

22. Gooyit M, Peng Z, Wolter WR, et al. A chemical biological strategy to facilitate diabetic wound healing. ACS Chem Biol. 2014;9(1): 105-110.

23. DeQuach JA, Mezzano V, Miglani A, et al. Simple and high yielding method for preparing tissue specific extracellular matrix coatings for cell culture. PLoS One. 2010;5(9):e13039.

24. Giner-Chavez BI, Van Soest PJ, Robertson JB, Lascano C, Reed JD, Pell AN. A method for isolating condensed tannins from crude plant extracts with trivalent ytterbium. J Sci Food Agric. 1997;74:359-368.

25. Trengove NJ, Stacey MC, MacAuley S, et al. Analysis of the acute and chronic wound environments: the role of proteases and their inhibitors. Wound Repair Regen. 1999;7(6):442-452.

26. Klebe RJ, Harriss JV. A technically simple "non-lethal" vital staining procedure for viral plaque and cell transformation assays. Brief report. Arch Virol. 1984;81(3-4):359-362.

27. Shaw JE, Sicree RA, Zimmet PZ. Global estimates of the prevalence of diabetes for 2010 and 2030. Diabetes Res Clin Pract. 2010;87(1): 4-14.

28. Loryman C, Mansbridge J. Inhibition of keratinocyte migration by lipopolysaccharide. Wound Repair Regen. 2008;16(1):45-51.

29. Saarialho-Kere UK. Patterns of matrix metalloproteinase and TIMP expression in chronic ulcers. Arch Dermatol Res. 1998;290 Suppl: S47-S54.

30. Black J, Baharestani M, Cuddigan J, et al; National Pressure Ulcer Advisory Panel. National Pressure Ulcer Advisory Panel's updated pressure ulcer staging system. Dermatol Nurs. 2007;19(4):343-349.

31. Schlie-Wolter S, Ngezahayo A, Chichkov BN. The selective role of ECM components on cell adhesion, morphology, proliferation and communication in vitro. Exp Cell Res. 2013;319(10):1553-1561.

32. Serra R, Buffone G, Falcone D, et al. Chronic venous leg ulcers are associated with high levels of metalloproteinases- 9 and neutrophil gelatinaseassociated lipocalin. Wound Repair Regen. 2013;21(3):395-401.

33. Toriseva M, Laato $M$, Carpén $O$, et al. MMP-13 regulates growth of wound granulation tissue and modulates gene expression signatures involved in inflammation, proteolysis, and cell viability. PLoS One. 2012;7(8):e42596.

34. Brandner JM, Zacheja S, Houdek P, Moll I, Lobmann R. Expression of matrix metalloproteinases, cytokines, and connexins in diabetic and nondiabetic human keratinocytes before and after transplantation into an ex vivo wound-healing model. Diabetes Care. 2008;31(1):114-120.

35. Amato B, Coretti G, Compagna R, et al. Role of matrix metalloproteinases in non-healing venous ulcers. Int Wound J. Epub October 24, 2013.
36. Gill SE, Parks WC. Metalloproteinases and their inhibitors: regulators of wound healing. Int J Biochem Cell Biol. 2008;40(6-7):1334-1347.

37. Lobmann R, Zemlin C, Motzkau M, Reschke K, Lehnert H. Expression of matrix metalloproteinases and growth factors in diabetic foot wounds treated with a protease absorbent dressing. J Diabetes Complications. 2006;20(5):329-335.

38. Serra R, Gallelli L, Buffone G, et al. Doxycycline speeds up healing of chronic venous ulcers. Int Wound J. Epub April 5, 2013.

39. Serra R, Grande R, Buffone G, Gallelli L, De Franciscis S. The effects of minocycline on extracellular matrix in patients with chronic venous leg ulcers. Acta Phlebologica. 2013;14(3):99-107.

40. Dalla Paola L. Diabetic foot wounds: the value of negative pressure wound therapy with instillation. Int Wound J. 2013;10 Suppl 1: 25-31.

41. Druecke D, Lamme EN, Hermann S, et al. Modulation of scar tissue formation using different dermal regeneration templates in the treatment of experimental full-thickness wounds. Wound Repair Regen. 2004;12(5):518-527.

42. Vivas A, Escandon J, Lebrun E, Choudhary S, Tang J, Kirsner RS. New therapies for treatment of diabetic foot ulcers: a review of current clinical trials. Surg Technol Int. 2010;20:83-96.

43. Shrivastava R, inventor; Naturveda, applicant. New synergic association for the treatment of deep skin or mucosa injuries. Patent WO2010045969 A1. April 29, 2010

44. Shrivastava R, inventor; Vitro-Bio Sarl, applicant. New synergistic compositions for the treatment of topical viral infections. Patent WO2011082835 A1. July 14, 2011.

45. Tixier JM, Godeau G, Robert AM, Hornebeck W. Evidence by in vivo and in vitro studies that binding of pycnogenols to elastin affects its rate of degradation by elastases. Biochem Pharmacol. 1984;33(24): 3933-3939.

46. Shrivastava R. A new therapeutic approach to neutralize throat surface proteases and virus glycoproteins simultaneously for the treatment of influenza virus infection. International Journal of Virology. 2011;7(2): 53-63.

47. Shrivastava R. A pilot clinical trial to evaluate the efficacy of a topical antiviral osmotically active hypertonic solution for the treatment of influenza virus induced sore throat. J Clin Trials. 2011;102(1):1-7.

48. Shrivastava R, Deshmukh S, Rousse M. Comparison of a hypertonic tannin-rich solution vs $3 \% \mathrm{NaCl}$ solution as treatment for rhinosinusitis. American Journal of Pharmtech Research. 2013;3(2):1-9.

49. Le Bourvellec C, Renard CM. Interactions between polyphenols and macromolecules: quantification methods and mechanisms. Crit Rev Food Sci Nutr. 2012;52(3):213-248.

50. Serban MA, Prestwich GD. Modular extracellular matrices: solutions for the puzzle. Methods. 2008;45(1):93-98.
Chronic Wound Care Management and Research

\section{Publish your work in this journal}

Chronic Wound Care Management and Research is an international, peer reviewed, open access, online journal publishing original research, reviews, editorials, and commentaries on the causes and management of chronic wounds and the major issues related to chronic wound management. Topics also include chronic wounds as comorbidities to other
Dovepress

conditions, patient adherence to therapy, and the economic burden of chronic wounds. The manuscript management system is completely online and includes a very quick and fair peer review system, which is all easy to use. Visit http://www.dovepress.com/testimonials.php to read real quotes from published authors. 